\title{
La opinión de expertos \\ como instrumento para evaluar la inversión en educación primaria
}

\section{Ernesto Schiefelbein}

Rector, Universidad Santo Tomás

Santiago de Chile

eschief@ust.cl

\section{Laurence Wolff}

Banco Interamericano

de Desarrollo

larryw@iadb.org

\section{Paulina Schiefelbein}

Centro de Investigación

y Desarrollo

de la Educación (CIDE),

Santiago de Chile
La mayoría de las inversiones educacionales se basan en conjeturas parcialmente probadas o en simples hipótesis sobre la relación costo/efectividad de una intervención dada. En realidad, sólo se ha contado con estimaciones de la rentabilidad media de cada tipo de educación, a pesar de que éstas sean muy diferentes de las marginales. En este artículo se describe un nuevo enfoque para evaluar la relación costo/efectividad de las inversiones educativas. Los autores solicitaron la opinión de diez investigadores educacionales de prestigio mundial sobre el impacto que tendría en los logros de aprendizaje de los alumnos un conjunto de cuarenta intervenciones que suelen ser consideradas deseables para mejorar la educación primaria, y complementaron las respuestas recibidas con cálculos propios del costo de cada una de ellas con el propósito de establecer un índice de su costo-efectividad. Sobre esta base, concluyeron que los proyectos educativos puestos en marcha en la región no han incluido muchas de las intervenciones identificadas como más eficientes, lo que ha limitado la calidad de la educación impartida y su posible contribución al éxito económico, a pesar del aumento considerable de las inversiones en educación realizadas por los gobiernos y los organismos internacionales en los años noventa. El artículo finaliza con varias recomendaciones orientadas a corregir esta situación, considerando simultáneamente el impacto y el costo de las diferentes intervenciones educativas. 


\section{La baja calidad de la educación en América Latina}

En América Latina, determinar cuál es la forma más eficiente de utilizar los limitados recursos de sus escuelas adquiere ribetes críticos. La calidad de la educación en la región latinoamericana es muy inferior a la de los países con los que compite, tanto en términos cuantitativos (tasa de alumnos graduados y nivel educativo medio de la fuerza laboral) como en el grado de utilización de sus conocimientos y aprendizaje (OCDE, 2000; UNESCO, 2000). Recientemente, el Laboratorio Latinoamericano de Evaluación de la Calidad de la Educación, de UNESCO/OREALC, ha difundido información comparativa sobre el aprendizaje en $3^{\circ}$ y $4^{\circ}$ grado de enseñanza primaria en la región. El cuadro 1 muestra los puntajes obtenidos por once países latinoamericanos en la sección de matemáticas de una prueba regional de la UNESCO, que mide habilidades mucho más simples y menos sofisticadas que las pruebas apli-

\begin{tabular}{llc} 
CUADRO 1 & \multicolumn{2}{l}{$\begin{array}{l}\text { América Latina (once países): Puntajes } \\
\text { medios en la prueba regional de la } \\
\text { UNESCo para matemáticas de }\end{array}$} \\
& ${ }^{\circ}$ grado ${ }^{\text {a }}$ \\
\hline País & Puntaje \\
\hline Cuba & 353 \\
Argentina & 269 \\
Brasil & 269 \\
Chile & 265 \\
Colombia & 258 \\
México & 256 \\
Paraguay & 248 \\
Bolivia & 245 \\
República Dominicana & 234 \\
Venezuela & 231 \\
Honduras & 226
\end{tabular}

Fuente: UNESCO, 1998.

a Puntajes medios estandarizados de 250 para $3^{\circ}$ y $4^{\circ}$ grado y desviación estándar de 50 . El alumno medio de la región contestó en forma correcta aproximadamente el 50\% de las preguntas; el estudiante cubano respondió el $85 \%$ de ellas.

$\square$ Los lectores que deseen aplicar la metodología descrita en este documento deben solicitar la autorización de los autores a través de los siguientes correos electrónicos: Larryw@iadb.org; o eschief@ust.cl. cadas en los países industrializados. Vemos que, salvo en Cuba, los rendimientos son deficientes.

Los resultados de la prueba también revelan que el rendimiento de las zonas rurales está por debajo del de las zonas urbanas (salvo en Colombia), que las capitales superan el puntaje de las ciudades menores y que las escuelas privadas (excepto en la República Dominicana) obtienen mejores resultados que las escuelas públicas.

En el ramo de castellano los rendimientos son similares. Los alumnos de la mitad de la población con ingresos inferiores al promedio contestan correctamente alrededor del $40 \%$ de las preguntas. Como las pruebas ofrecen cuatro alternativas, un alumno que sepa las respuestas correctas de un $20 \%$ de las preguntas también contestará "correctamente" (pero por azar) una de cada cuatro de las otras 80 preguntas cuyas respuestas correctas no conoce. Con éstas completa el $40 \%$ de respuestas correctas. Esto quiere decir que la mayoría de los alumnos no entiende los mensajes de los textos escritos que se les presentan en esa prueba. En otras palabras, la gran mayoría de los futuros trabajadores son analfabetos funcionales.

Los malos resultados obtenidos obligan a examinar cuidadosamente los factores críticos que suelen considerarse determinantes del nivel de aprendizaje y de retención. Entre ellos es frecuente mencionar la disponibilidad y uso de libros de texto, la provisión de educación preescolar, la instrucción por radio y algunos programas de capacitación en el trabajo (Lockheed y Verspoor, 1991), pese a que sus efectos pocas veces se han calculado y nunca se han asociado a sus costos. Hasta 1998, fueron escasos los experimentos realizados al nivel de la educación primaria en América Latina que recibieron una adecuada evaluación y difusión. Entre ellos mencionaremos: matemáticas por radio en Nicaragua (Jamison, Searle, Heyneman y Galda, 1981); televisión educativa en El Salvador (Hornik, Ingle, Macanany y Schramm, 1973); Programa de Educación del Nordeste en Brasil (Harbison y Hanushek, 1992); Escuela Nueva en Colombia (McEwan, 1995; Psacharopoulos, Rojas y Vélez, 1995; Rojas y Castillo, 1998); las Escuelas P-900 en Chile (Gutman, 1993); Escuelas Fe y Alegría (Swope y Latorre, 1998); EDUCO en 
El Salvador (El Salvador, Ministerio de Educación, 1996); y las escuelas primarias aceleradas de Brasil (Araujo Oliveira, 1998). Con el tiempo, gracias al mayor número de programas nacionales de medición de logros de aprendizaje, será posible determinar cuáles intervenciones han tenido un efecto significativo sobre el aprendizaje. ${ }^{1}$

La escasa información disponible sobre los factores que influyen en los resultados del aprendizaje, ${ }^{2} \mathrm{a}$ pesar de su importancia, así como las dificultades inherentes a las investigaciones tradicionales de eficiencia, llevó a que los autores buscaran una estrategia diferente para ayudar a investigadores y educadores profesionales a adquirir una mayor comprensión de los aspectos fundamentales del tema y desarrollaran un instrumento que tal vez facilite la capacitación, la creación de consenso y la identificación de áreas críticas de investigación.

\section{II}

\section{Cuarenta estrategias usadas habitualmente} para mejorar la calidad de la educación en América Latina

Para obtener respuestas comparables del grupo de diez expertos internacionales, activos en universidades y organismos internacionales, cuya opinión se solicitó, los autores diseñaron un índice de costo-efectividad (eficiencia) que definía con precisión cada una de las posibles intervenciones cuyo impacto y factibilidad se

\footnotetext{
${ }^{1}$ Véase un resumen detallado de la experiencia reciente en Rojas y Esquivel (1998).

${ }^{2}$ Además de las investigaciones mencionadas, se han hecho otros esfuerzos meritorios en este sentido. Los estudios sectoriales de Honduras y El Salvador sobre repetición y fracaso escolar (Reimers y McGinn, 1997) han llevado a implementar una serie de programas orientados a reducir la repetición. Sin embargo, las escasas iniciativas de investigación sistemática que se han realizado, en particular las relativas al impacto sobre el aprendizaje, se encuentran plagadas de incertidumbres e inconsistencias. En el caso de la descentralización concretada en El Salvador a través del programa EDUCO se ha comprobado un aumento de la matrícula escolar rural y mejoras en términos de logros de aprendizaje y tasas de retención, pero se observan pocas diferencias claras entre el nivel de aprendizaje de las escuelas EDUCO y el de las escuelas salvadoreñas tradicionales. Las escuelas EDUCo disponen de más insumos, dedican más tiempo a la instrucción y han intensificado la participación de los padres. Sin embargo, es posible que el mejoramiento de los logros de aprendizaje sea el resultado del mayor nivel de expectativas que acompaña a la descentralización, que por cierto incluye un enfoque más orientado al aprendizaje (Meza, 1997). También se observa que las escuelas autónomas de Nicaragua exhiben tasas más altas de retención, si bien otros factores pueden estar influyendo en esta medición. Se aprecia un mayor grado de satisfacción entre docentes y padres, a pesar de que los resultados escolares tampoco son claros (Castillo, 1998). De hecho, si bien existen sólidas razones políticas y sociales que avalan la adopción de un régimen descentralizado - en particular el fortalecimiento de la sociedad civil—, a nivel mundial existe escasa evidencia de que la descentralización tenga como corolario un aumento del aprendizaje.
}

pedía evaluar, con el fin de reducir la variación por diferencias en el alcance "supuesto" de cada una de ellas. Los entrevistados debían estimar el impacto que cada una de 40 intervenciones en materia de educación primaria consideradas posibles (recuadro 1) podría tener sobre los logros de aprendizaje (medidos por los puntajes en una prueba estandarizada que se administraría a fines del sexto grado), y a continuación, debían estimar la probabilidad (porcentual) de una implementación exitosa. Los autores, a su vez, incorporaron sus propios cálculos sobre los costos unitarios de las 40 intervenciones. Con estos tres antecedentes se calculó el índice de costo-efectividad de cada una de ellas.

Las 40 intervenciones presentadas a los expertos, que afectan a las doce áreas operacionales del sistema educativo incluidas en el cuadro 2, se seleccionaron sobre la base de siete tipos diferentes de criterios:

i) los componentes de programas y proyectos educacionales aplicados en América Latina en los últimos 20 años, exitosos o no;

ii) las prioridades y recomendaciones propuestas por los organismos internacionales y los bancos de desarrollo (Lockheed y Verspoor, 1991; Banco Mundial, 1994; Carnoy y Castro, 1997);

iii) las principales conclusiones derivadas de las encuestas diagnósticas regionales realizadas en los años noventa (Schiefelbein, coord., 1998; Wolff, Schiefelbein y Valenzuela, 1994);

iv) el análisis de estudios previos de investigación sobre el costo-efectividad de estrategias claves (Wolff, 


\section{Recuadro 1}

Cuarenta intervenciones educativas posibles para América Latina

1. Aplicar una política que prohíba cambiar de curso a los profesores durante el año escolar.

2. Implementar una política que ubique los mejores maestros en el primer grado.

3. Vigilar que se cumpla la norma sobre la duración oficial del año escolar.

4. Prolongar el horario escolar en una hora diaria (40 minutos de clases, 20 minutos de actividades recreativas) y pagar al maestro el salario proporcional adicional.

5. Prolongar el año escolar en una semana y pagar al maestro el salario proporcional adicional.

6. Otorgar a los maestros de escuelas rurales un aumento equivalente al 50\% del sueldo, como estrategia para captar a profesionales mejor capacitados y elevar el porcentaje de maestros titulados o acreditados.

7. Aumentar el sueldo de los docentes en un $10 \%$ en términos reales, con el compromiso de no declararse en huelga por un período de dos años.

8. Aumentar el sueldo de los docentes en un $20 \%$ en términos reales, con el compromiso de no declararse en huelga por un período de tres años.

9. Despedir a la mitad del personal educacional que ocupa cargos burocráticos (partida que actualmente representa el 5\% del costo unitario) y crear una nueva burocracia altamente capacitada y motivada que perciba, en promedio, 2.1 veces su salario previo.

10. Crear un sistema de información para la gestión (SIG) con el objeto de identificar a las escuelas de menor rendimiento e informar a sus supervisores.

11. Descentralización: autorizar a los rectores a administrar fondos y a despedir y contratar maestros contando solamente con la aprobación de un consejo local, sin aumentar la capacidad del ministerio de educación para evaluar o fiscalizar dichas facultades.

12. Igual que el punto anterior, salvo que la capacidad del ministerio se incrementa en forma significativa.

13. Administrar una prueba de matemáticas y lectura a una muestra del $10 \%$ de los alumnos de $4^{\circ}$ grado y entregar los resultados (numéricos) a todos los maestros de ese nivel.

14. Tal como en el caso anterior, pero analizando los resultados de la muestra, proponiendo las acciones correctivas necesarias y organizando seminarios de seguimiento para los maestros de $4^{\circ}$ grado (una semana).

15. Administrar una prueba a la totalidad de los alumnos de $4^{\text {to }}$ grado (igual que la de los dos puntos anteriores).

16. Proporcionar a cada alumno un texto estándar de matemáticas y uno de lectura (cada uno de aproximadamente 200 páginas) con su correspondiente manual para el profesor, sin capacitar al maestro en su uso.

17. Lo mismo que el punto anterior, aunque esta vez capacitando al maestro (una semana por año).

18. Elaborar un conjunto de materiales didácticos en lectura y matemáticas para instrucción personalizada y distribuirlos entre los alumnos (400 páginas por alumno, renovables cada tres años).

19. Dotar a cada aula de una pequeña biblioteca (100 libros), renovable cada cinco años.

20. Programa de alimentación escolar: colación para todos (un vaso de leche y pan) distribuida en forma gratuita.

21. Programa de alimentación escolar: colación (un vaso de leche y pan) distribuida en forma gratuita a la mitad de los niños; el resto debe pagar.

22. Programa de alimentación escolar: almuerzo gratis para todos.

23. Programa de alimentación escolar: almuerzo gratis para la mitad de los niños; el resto debe pagar.

24. Examen médico anual y derivación a especialistas o centros especializados. No incluye el tratamiento médico que prestaría el sistema de salud.

25. Examen oftalmológico realizado en la escuela y derivación a especialistas o centros especializados. No incluye los tratamientos.

26. Adecuar y transmitir programas televisivos de gran prestigio a la población preescolar, por ejemplo, Plaza Sésamo (250 programas por año). Sólo para ser visto en el hogar.

27. Campaña por los medios de comunicación para que los padres den estimulación temprana al niño (¿Se acordó anoche de leerle una página a su niño?), a través de 30 avisos publicitarios de un minuto cada uno, durante una semana. 
28. Un año de atención preescolar para el desarrollo del 50\% de los niños en situación de riesgo a un costo unitario equivalente al de un año de primaria.

29. Igual que el punto anterior a un costo unitario equivalente a medio año de primaria.

30. Un año de cuidado de preescolares sin contenido de desarrollo educacional (costo unitario equivalente a medio año de primaria).

31. Dar capacitación general en servicio a los profesores (perfeccionamiento) cuatro semanas al año (sin material de seguimiento para utilizar en clase).

32. Capacitación en servicio (una semana al año), de carácter práctico y focalizado en el desarrollo de estrategias de aprendizaje cooperativo en el aula (trabajo grupal) y en el uso activo del tiempo del estudiante

33. Capacitación focalizada, en el uso de material didáctico programado (una semana).

34. Capacitación focalizada, orientada a familiarizar al maestro con los objetivos y estrategias del currículo moderno (una semana), al estilo del programa CENAMEC de Venezuela.

35. Crear un programa de subsidio gubernamental destinado a mejorar la calidad de la formación docente inicial, con miras a enfrentar los desafíos del siglo XXI. El gobierno aporta 50 dólares adicionales (por alumno) a las instituciones formadoras de docentes que modifican sus programas para dar importancia al aprendizaje activo, la excelencia, el compromiso y la responsabilidad.

36. Modificar el programa de estudios en las áreas de lectura y matemáticas, con la colaboración de especialistas locales, y hacer llegar una copia a cada maestro (sin darles capacitación en servicio o estudio en terreno del currículo implementado).

37. Preparar e implementar un currículo bilingüe para $1^{\mathrm{er}}$ y $2^{\circ}$ grados en las áreas de lectura y matemáticas, que incluya material didáctico, capacitación y selección de docentes, así como la adaptación y traducción de los libros de texto.

38. Elaborar e implementar programas educativos interactivos para español y matemáticas (con sus correspondientes materiales de enseñanza/aprendizaje) y transmitirlos por radio a toda la población escolar.

39. Dar a todos los estudiantes de primaria acceso a computadoras durante una hora a la semana, con el fin de estudiar LOGO.

40. Crear un consenso nacional sobre la importancia de mejorar la educación básica. Luego, enviar un paquete didáctico completo a todas las escuelas en situación de riesgo (el 50\% de las escuelas de rendimiento más bajo) que contemple: materiales de autoaprendizaje, capacitación en técnicas de aprendizaje cooperativo y activo, talleres de carácter práctico, participación de la comunidad, administración basada en la escuela, evaluación formativa y modalidades sistemáticas de pruebas (testing) y de retroalimentación.

Schiefelbein y Valenzuela, 1994; Lockheed y Verspoor, 1991; Verspoor, 1989);

v) las investigaciones sobre factores que afectan al rendimiento (Fuller y Clarke, 1994);

vi) la experiencia de reforma llevada a cabo en California (Chrispeels, 1997), y

vii) la posibilidad de expresar esta información en forma simple y precisa de manera que la comparación de las estimaciones sea confiable.

Las intervenciones que se identificaron incluyeron las cinco "promisorias intervenciones de política" seleccionadas por Lockheed y Verspoor (1991, p. 28): tiempo de instrucción; libros de texto y materiales didácticos; aumento de la capacidad de aprendizaje del alumno (alimentación, salud y educación inicial); capacitación docente, y currículo. Se incluyeron además algunas intervenciones que predominan en la región,
CUADRO 2

\section{Intervenciones educativas por áreas operacionales del sistema educativo}

\begin{tabular}{ll}
\hline Area operacional & $\begin{array}{l}\text { Número de la } \\
\text { intervención }\end{array}$ \\
\hline Tiempo dedicado a la tarea & $3,4,5$ \\
Gestión académica & 1,2 \\
Sueldos & $6,7,8$ \\
Administración y descentralización & $9,10,11,12$ \\
Administración de pruebas (testing) & $13,14,15$ \\
Libros de texto y materiales de autoaprendizaje & $16,17,18,19$ \\
Alimentación y salud & $20,21,22,23,24,25$ \\
Educación inicial & $26,27,28,29,30$ \\
Capacitación de los docentes & $31,32,33,34,35$ \\
Currículo & 36,37 \\
Radio y computadoras & 38,39 \\
Conjunto de intervenciones & 40
\end{tabular}

a Los números de cada intervención corresponden a los asignados a ellas en el recuadro 1 , donde se describen en detalle. 
a pesar de que había evidencias de su ineficacia. Una primera versión de estas 40 estrategias se ensayó con altos funcionarios de los países que participaron en tres cursos de planificación organizados por la UNESCO en 1994 y 1996. Como resultado de esta aplicación se definieron, finalmente, las cuarenta intervenciones que se describen en el recuadro 1.

El público suele creer (y el razonamiento pedagógico apoyar) que las combinaciones de intervencio- nes pueden tener un efecto acumulativo, razón por la cual varias estrategias combinan dos o más intervenciones. Por ejemplo, además de la intervención 11 (descentralizar la autoridad de los rectores de escuelas), se incluye la 12 (igual a la anterior, pero aumentando la capacidad de fiscalización otorgada al ministerio), o bien a la intervención 16 (proporcionar dos libros a cada alumno) se suma la 17 (igual a la anterior, más una semana de capacitación docente).

\section{III}

\section{El país, "Concordia", en el que se aplicarían las estrategias}

Con el fin de generar comparaciones válidas, se definió un país beneficiario: "Concordia". Dicho país prototipo, que se describe en el recuadro 2 , se construyó sobre la base de valores medios observados en América Latina que incluyen características demográficas, niveles de costo, razón estudiantes por maestro, insumos escolares y puntajes de respuestas correctas en las pruebas. En consecuencia, todas las respuestas (de los expertos mundiales y los planificadores) están vinculadas a un contexto educacional común.

Era necesario contar con este "constructo artificial" sin historia ni contexto, ya que el costo-efectivi- dad fluctuará significativamente según el número de estudiantes que conformen el sistema educacional de cada país, el grado de desarrollo educacional del mismo y su producto interno bruto por habitante. Por ejemplo, los costos relativos de los insumos pueden ser bastante más elevados en un país donde el costo medio unitario de la educación primaria es de 100 dólares o menos, comparado con el promedio regional de 200 dólares. Lo mismo sucedería en los países más pequeños donde los costos fijos son elevados y los costos variables son bajos (por ejemplo, evaluaciones basadas en muestras y educación a distancia).

\section{Recuadro 2 \\ Concordia}

- Población: 20 millones

Población rural: $30 \%$

Población indígena: $10 \%$

- Porcentaje de alumnos que ha completado la educación primaria (seis años): $60 \%$

- Razón estudiantes por maestro: 29:1

- Costo unitario de la educación primaria: 200 dólares

- Matrícula en el nivel primario (grados $1^{\circ}$ a $6^{\circ}$ ): 2 millones

- Costo total del sistema de educación primaria: 400 millones de dólares

- Porcentaje del presupuesto destinado a pagar el sueldo del magisterio: $90 \%$

- Calendario escolar: cuatro horas diarias y 27 sesiones semanales de clases de 45 minutos cada una

- El $50 \%$ de los estudiantes tiene (o usa) los libros de texto básicos

- No existe un sistema de evaluación. Sin embargo, a finales de sexto grado, se administró una prueba a una muestra reducida de alumnos. La prueba se ajustó al currículo oficial de matemáticas y español. El puntaje promedio de la prueba fue de 50 respuestas correctas sobre 100 puntos posibles. Este puntaje correspondería a un buen dominio de las materias contenidas en los currículos. 


\section{IV}

\section{Características del panel de expertos mundiales}

Los autores seleccionaron expertos que reunían seis características: i) haber publicado artículos en buenas revistas profesionales; ii) haber sido mencionados reiteradamente por planificadores y profesionales de la educación; iii) tener fácil acceso a los resultados obtenidos por investigaciones de reciente data; iv) haber participado en proyectos en varios países de América Latina; v) ser líderes en el análisis de iniciativas de desarrollo educacional, y vi) tener experiencia laboral con organismos multilaterales de desarrollo en la región. Además, se buscó un equilibrio entre el número de expertos de América del Norte y de América Latina.

Los nombres del panel de expertos mundiales y las instituciones en que trabajan evitan mayores comentarios sobre el peso de sus opiniones: Martin Carnoy y Henry Levin (Universidad de Stanford); Noel McGinn y Fernando Reimers (Universidad de Harvard); Claudio de Moura Castro (Banco Interamericano de Desarro1lo); Steve Heyneman, Himelda Martínez y Eduardo Velez (Banco Mundial); Jeffrey Puryear (Diálogo Interamericano) y Juan Carlos Tedesco (Oficina Internacional de Educación de Ginebra, UNESCO). Los autores desean expresar aquí su profundo agradecimiento al panel que apoyó este estudio.

La respuesta de los expertos fue entusiasta, dado su intenso interés en los temas planteados (sólo los dos representantes europeos de la muestra original no pudieron contestar la encuesta). No se apreciaron diferencias sistemáticas en las estimaciones entregadas por los expertos de las dos regiones indicadas más arriba.

V

\section{El indicador del rendimiento académico de los alumnos}

Se seleccionó como variable dependiente el "puntaje obtenido por el alumno en una prueba estandarizada rendida a finales del $6^{\circ}$ grado". Se supuso que esta prueba sería similar a las pruebas de idioma y matemáticas administradas por la UNESCO/OREALC a 13 países en 1997, en las cuales el promedio de alumnos contestó correctamente el 50\% de las preguntas (cuadro 1). Las preguntas de esas pruebas representaban una opinión consensuada de los países participantes acerca de lo que se esperaría de un currículo común. En este caso, las pruebas referidas a criterios anticipan un $100 \%$ de respuestas correctas, puesto que esa es la exigencia que impone el currículo. Por lo tanto, con ayuda de la intervención (estrategia) apropiada, es factible que cierto número de estudiantes obtenga resultados más altos, como se demostró en el caso de Cuba.

Este enfoque no está exento de problemas. $\mathrm{Mu}$ chos niños abandonan la escuela antes de llegar al $6^{\circ}$ grado o repiten curso, especialmente en los países más pobres. Si la variable seleccionada hubiera sido el "porcentaje de niños que finaliza el $6^{\circ}$ grado", habría habido cambios en las estimaciones de los expertos, aunque no significativos. Por ejemplo, la alimentación escolar habría tenido un impacto mucho mayor en la retención del alumno que en el aprendizaje, puesto que se ha supuesto que constituye un aliciente para asistir a la escuela.

La utilización de los puntajes de pruebas como variable dependiente es más útil en aquellos sistemas educacionales en que se gradúan grandes contingentes de estudiantes del nivel primario (Costa Rica o Argentina), siendo mucho menos adecuada para los sistemas de educación primaria que exhiben altos porcentajes de desertores (Honduras o Guatemala).

En todo caso, casi todos los sistemas educacionales de la región avanzan rápidamente hacia los seis años de instrucción y, por lo tanto, hacer hincapié en la calidad de la educación es importante para casi todos los países. El puntaje general de una prueba de $6^{\circ}$ grado constituye una medida simple, aunque clara, de los actuales intentos por mejorar la calidad de la educación en América Latina. 


\section{VI}

\section{Estimación del impacto de las estrategias en el país prototipo}

Se les solicitó a los diez expertos mundiales que por cada intervención aplicada en "Concordia" proporcionaran las dos siguientes estimaciones: en primer lugar, el porcentaje medio de mejoramiento del rendimiento académico de los alumnos de $6^{\circ}$ grado que hasta ahora obtenían un puntaje de 50 puntos (de 100 posibles) en una prueba estandarizada de lectura y matemáticas, en comparación con un grupo de control que no se beneficiara de la intervención (cuadro 3, columna A), y en segundo lugar, la probabilidad (en porcentaje) de una plena implementación de la intervención, teniendo en cuenta consideraciones técnicas y políticas (cuadro 3, columna B).
Posteriormente, los autores realizaron una tercera estimación: la del probable aumento del costo unitario de operación originado por la intervención, incluyendo el gasto de inversión, ambos calculados en forma anual (véanse el anexo 1 y el cuadro 3, columna D).

Se consideró inapropiado solicitar a los expertos una estimación de los costos por el hecho de ser ésta una pregunta técnica de prolongado desarrollo y que, además, tiene una respuesta "correcta" (o que el lector puede modificar con los antecedentes de un país específico). Las estimaciones de costo tuvieron como patrón un país de la región, de tamaño e ingreso medios (ver las características de "Concordia"). En el anexo se presenta la justificación detallada del cálculo.
Número y descripción de la intervención en orden descendente de costo-efectividad ${ }^{\mathrm{a}}$

$\begin{array}{ccccc}\text { A } & \text { B } & \text { C } & \text { D } & \text { E } \\ \text { Aumento } & \text { Probabilidad } & \text { Impacto } & \text { Aumento } & \text { Costo } \\ \text { estimado del } & \text { de una } & \text { probable }(\%) & \text { estimado del } & \text {-efectividad } \\ \text { rendimiento } & \text { implementación } & {\left[\mathrm{A}^{*} \mathrm{~B}\right]} & {\text { costo }(\%)^{\mathrm{d}}}^{\mathrm{C}} & \text { [C/D] } \\ \text { académico }(\%)^{\mathrm{b}} & \text { adecuada }(\%)^{\mathrm{c}} & & & \end{array}$

$\mathrm{F}$

Costo unitario de aumentar el logro académico en un punto (dólares)

2. Asignar los mejores maestros al primer grado.

3. Exigir el efectivo cumplimiento de la duración oficial del año escolar

1. Prohibir el cambio de curso del profesor durante el año escolar

13. Prueba objetiva al $10 \%$ de los alumnos de $4^{\circ}$ grado y distribuir resultados entre los maestros

11. Descentralización

27. Campaña en los medios masivos para dar estimulación temprana y lectura en el hogar

10. SIG para identificar escuelas de bajo rendimiento

25. Examen oftalmológico en la escuela y derivar a especialistas

35. Subsidio (50 dólares por alumno) para mejor formación inicial del docente

14. Prueba objetiva al $10 \%$ de los alumnos de $4^{\circ}$ grado y ofrecer estrategias correctivas . (1 semana)

9. Reducir la mitad de la burocracia y pagar sueldos más altos

36. Revisar el currículo en áreas de matemáticas y lectura y distribuirlo

38. Instrucción interactiva a través de programas de radio

37. Elaborar e implementar un currículo bilingüe

15. Administrar pruebas a la totalidad de los estudiantes de $4^{\circ}$ grado

19.8

10.6

5.0

4.1

9.3

8.1

10.2

3.2

12.3

8.9

1.9

10.7

11.7

12.3
58.0

49.5

72.0

73.5

47.5

71.9

68.0

66.0

56.0

60.0

36.0

66.9

57.5

50.6

62.5
11.5

5.2

3.6

3.0

4.4

5.8

6.9

2.1

6.6

7.4

3.2

1.3

6.2

5.9

7.7
1531.2

0,003

0006

0008

0066

0068

0086

0,144

0189

0.212

0230

0,311

0315

0,350

0,356

0411 
Cuadro 3 (continuación)

\begin{tabular}{|c|c|c|c|c|c|c|}
\hline $\begin{array}{l}\text { Número y descripción de la intervención en orden } \\
\text { descendente de costo-efectividad }{ }^{\mathrm{a}}\end{array}$ & $\begin{array}{c}\text { A } \\
\text { Aumento } \\
\text { estimado del } \\
\text { rendimiento } \\
\text { académico }(\%)^{\mathrm{b}}\end{array}$ & $\begin{array}{c}\text { B } \\
\text { Probabilidad } \\
\text { de una } \\
\text { implementación } \\
\text { adecuada }(\%)^{\mathrm{c}}\end{array}$ & $\begin{array}{c}\mathrm{C} \\
\text { Impacto } \\
\text { probable }(\%) \\
{\left[\mathrm{A}^{* \mathrm{~B}]}\right.}\end{array}$ & $\begin{array}{c}\text { D } \\
\text { Aumento } \\
\text { estimado del } \\
\text { costo }(\%)^{\mathrm{d}}\end{array}$ & $\begin{array}{c}\text { E } \\
\text { Costo } \\
\text {-efectividad } \\
\text { [C/D] }\end{array}$ & $\begin{array}{c}\mathrm{F} \\
\text { Costo unitario de } \\
\text { aumentar el logro } \\
\text { académico en un } \\
\text { punto (dólares) }\end{array}$ \\
\hline
\end{tabular}

18. Dar material didáctico para instrucción personalizada

26. Transmitir buenos programas televisivos a la población preescolar

12. Descentralización con buena supervisión

16. Proporcionar libros de texto (estándar) para uso en clase

19. Dotar las salas de clases de pequeñas bibliotecas

17. Dar libros de texto (estándar) y capacitar al maestro en su uso

5. Prolongar una semana la duración del año escolar

32. Capacitar al maestro en el desarrollo de métodos de aprendizaje cooperativo

33. Capacitar al maestro en uso de textos de aprendizaje programado

34. Familiarizar al maestro con el currículo moderno

40. Intervención con un paquete de aprendizaje, administración local, capacitación y administración de pruebas

29. Programa preescolar orientado al desarrollo (50\% del costo unitario de primaria)

28. Programa preescolar orientado al desarrollo (100\% del costo unitario de primaria)

24. Examen médico anual y derivar a especialistas

30. Cuidado de preescolares sin asegurar su desarrollo educacional

6. Pagar a los maestros rurales un sobresueldo del $50 \%$

4. Prolongar el horario escolar en una hora diaria

7. Aumentar en $10 \%$ el sueldo del profesorado

21. Programa de alimentación escolar (50\% del alumnado recibe colación en forma gratuita)

8. Aumentar en $20 \%$ el sueldo del profesorado

20. Programas de alimentación escolar (un $100 \%$ del alumnado recibe colación en forma gratuita)

31. Capacitación de docentes en servicio sin material de seguimiento

23. Programas de alimentación escolar (50\% del alumnado recibe almuerzo gratuito)

39. Acceso de 1 hora por semana a trabajar con computadora

22. Programa de alimentación escolar (100\% del alumnado recibe almuerzo gratuito)

Promedios

\begin{tabular}{|c|c|c|c|c|c|}
\hline 16.5 & 72.5 & 12.0 & 1.5 & 8.0 & 0,500 \\
\hline 8.2 & 72.4 & 5.9 & 0.8 & 7.9 & 0505 \\
\hline 19.4 & 53.5 & 10.4 & 1.3 & 7.8 & 0,510 \\
\hline 11.5 & 74.5 & 8.6 & 1.5 & 5.7 & 0699 \\
\hline 8.5 & 76.5 & 6.5 & 1.4 & 4.7 & 0,846 \\
\hline 18.4 & 66.0 & 12.1 & 3.8 & 3.2 & 1235 \\
\hline 8.0 & 83.5 & 6.7 & 2.3 & 3.0 & 1347 \\
\hline 12.2 & 52.0 & 6.3 & 2.3 & 2.8 & 1419 \\
\hline 7.6 & 64.0 & 4.9 & 2.3 & 2.2 & 1850 \\
\hline 7.0 & 64.0 & 4.5 & 2.3 & 2.0 & 2009 \\
\hline 26.8 & 45.0 & 12.1 & 7.0 & 1.7 & 2322 \\
\hline 13.0 & 54.5 & 7.1 & 4.2 & 1.7 & 2354 \\
\hline 18.3 & 51.5 & 9.4 & 8.3 & 1.1 & 3538 \\
\hline 4.1 & 61.5 & 2.5 & 2.4 & 1.1 & 3,807 \\
\hline 5.7 & 65.9 & 3.8 & 4.2 & 0.9 & 4441 \\
\hline 18.6 & 65.0 & 12.1 & 13.5 & 0.9 & 4,467 \\
\hline 17.0 & 67.0 & 11.4 & 15.0 & 0.8 & 5268 \\
\hline 6.3 & 72.5 & 4.6 & 9.0 & 0.5 & 7882 \\
\hline 5.1 & 63.0 & 3.2 & 6.8 & 0.5 & 8403 \\
\hline 10.7 & 74.5 & 8.0 & 18.0 & 0.4 & 9032 \\
\hline 5.6 & 74.5 & 4.2 & 13.5 & 0.3 & 12943 \\
\hline 4.1 & 63.5 & 2.6 & 10.0 & 0.3 & 15364 \\
\hline 6.9 & 59.0 & 4.1 & 18.0 & 0.2 & 17686 \\
\hline 4.4 & 51.5 & 2.3 & 14.9 & 0.2 & 26337 \\
\hline 8.1 & 67.5 & 5.5 & 36.0 & 0.2 & 26337 \\
\hline 103 & 628 & 65 & 5.1 & 769 & 4,1 \\
\hline
\end{tabular}

a Véanse las descripciones detalladas de las intervenciones en el recuadro 1.

b Mejoramiento porcentual medio esperado en el rendimiento académico de alumnos de $6^{\circ}$ grado — con puntaje inicial de 50 puntos (de 100 posibles) - en una prueba estandarizada de lectura y matemáticas, en comparación con el grupo de control que no se benefició de la intervención .

c Probabilidad (porcentual) de una adecuada implementación de la intervención, basada en consideraciones técnicas y políticas.

d Probable aumento anual del costo unitario de operación originado por la intervención, incluyendo el gasto de inversión proyectado anualmente. 


\section{VII}

\section{Cálculo del índice de costo-efectividad}

Con las estimaciones descritas se elaboró un índice de costo-efectividad para cada intervención, que se calculó considerando lo siguiente:

$\mathrm{a}=$ porcentaje de la población escolar beneficiada por la intervención;

$\mathrm{b}=$ suponiendo que la intervención se implementa plenamente, porcentaje de aumento esperado en los puntajes de la prueba de la población beneficiaria;

$\mathrm{c}=$ probabilidad porcentual de una plena implementación de la intervención, y

$\mathrm{d}=$ porcentaje de aumento del costo anual de operación para la población beneficiaria.
Para la población beneficiaria I (Indice) $=\mathrm{b}^{*} \mathrm{c} / \mathrm{d}$; para el conjunto de la población tanto los costos como los efectos se ven proporcionalmente reducidos, si bien el valor del índice permanece inalterado (por ejemplo, $\mathrm{I}=\mathrm{b} * \mathrm{c} * \mathrm{a} / \mathrm{d} * \mathrm{a})$.

El cuadro 3 muestra, en orden descendente, los índices de eficiencia de las 40 intervenciones consideradas. Pero hay muchas formas de resumir la información de este cuadro y de sacar conclusiones, que incluyen el aumento porcentual esperado en los puntajes de pruebas, el aumento de puntajes en función de la factibilidad de implementar la intervención, y el costo-efectividad.

\section{VIII}

\section{Costo por unidad de impacto}

Es conveniente, a veces, comparar el costo que tiene cada intervención para elevar en uno por ciento el rendimiento académico. Este "costo por unidad de impacto (K)" es igual al costo total anual dividido por el impacto probable. En este caso: $K=d^{*} 200 /(50 * b * c)$. En la fórmula, 200 es el valor del costo unitario medio en dólares (recuadro 2) y 50 es el promedio de respuestas correctas en la prueba.
Los resultados se presentan en la columna $\mathrm{F}$ del cuadro 3. Son proporcionales al índice de costo-efectividad y toman en cuenta las distintas ponderaciones que resultan de considerar las dos constantes $(200 \mathrm{y}$ $50)$.

Dada esta característica el análisis de los resultados se hace solamente para el índice de costo-efectividad.

\section{IX}

\section{Análisis de los principales resultados}

Se han identificado las seis intervenciones que, a juicio de los expertos, tendrían el mayor impacto en el aprendizaje al ser implementadas con éxito (cuadro 4).

Como se esperaba, ocupa el primer lugar un enfoque basado en "sistemas" que integran una gran variedad de intervenciones: ésta es, por amplio margen, la estrategia señalada como la que produce los mayores efectos esperados, si se implementa apropiadamente. Pero es interesante observar que las cinco estrategias siguientes son "singulares" y producen un efecto muy similar en magnitud. Estas incluyen la asignación de los mejores maestros a primer grado, la adopción de un régimen descentralizado combinado con el reforzamiento de la autoridad central, la asignación a maestros rurales de un aumento sustancial de sueldo, la provisión de libros de texto estandarizados y capacitación en su uso y la oferta de programas preescolares orientados al desarrollo. 
CUADRO 4

\section{Las seis intervenciones de más alto impacto en la población beneficiaria cuando pueden ser implementadas con éxito \\ (En porcentajes)}

Intervención

Aumento esperado en los puntajes de pruebas

40. Intervenciones múltiples mediante un paquete didáctico con administración descentralizada para la escuela; capacitación y administración de pruebas

2. Asignar a los mejores maestros al primer grado

6. Pagar al maestro rural un sobresueldo del orden del $50 \%$

17. Dar libros de texto estándares y capacitar a los docentes en su uso

28. Programa preescolar orientado al desarrollo (100\% del costo de la escuela primaria)

CUADRO 5

Las seis intervenciones de más alto
impacto en la población beneficiaria,
considerando la factibilidad de su
implementación
(En porcentajes)

Intervención

Aumento esperado en los puntajes de pruebas

17. Proporcionar libros de texto estándares y capacitar a los docentes en su uso

40. Intervenciones múltiples mediante un paquete didáctico, administración descentralizada en la escuela; capacitación y administración de pruebas

6. Pagar al maestro rural un sobresueldo del orden del $50 \%$

18. Proporcionar material didáctico para la instrucción personalizada

2. Asignar a los mejores maestros al primer grado 11.5

4. Extender el horario escolar en una hora diaria 11.4

Sin embargo, los expertos manifestaron su preocupación ante la dificultad de llevar a la práctica estos enfoques, particularmente el basado en sistemas (columna B del cuadro 3). Cuando se toma simultáneamente en cuenta el efecto esperado en el aprendizaje y la probabilidad de una implementación adecuada (se multiplican las dos estimaciones) el probable impacto cambia considerablemente (columna $\mathrm{C}$ del cuadro 3 ). El cuadro 5 muestra las seis estrategias que alcanzan entonces los mayores impactos.
Las seis mejores intervenciones ahora incluyen la ampliación del horario escolar en una hora y la provisión de paquetes de aprendizaje personalizado. Se puede apreciar que, una vez consideradas las dificultades de aplicación, el enfoque basado en sistemas no es más eficiente que proporcionar una serie de insumos en forma separada. Además, los programas preescolares y la descentralización, por ser difíciles de llevar a la práctica, pierden su lugar entre las seis mejores intervenciones.

Pero la efectividad de las estrategias tiene que compararse en relación con un incremento similar del costo. Al dividir el incremento probable (neto) del rendimiento por el aumento del costo (columna $\mathrm{E}$ del cuadro 3) es posible, finalmente, identificar las estrategias más deseables. El cuadro 6 muestra las seis intervenciones que obtuvieron el índice general de costo-efectividad más alto.

Las intervenciones que alcanzan el índice de costo-efectividad más alto calculado son diferentes de aquellas que se espera tengan el más alto impacto, ya que existen varias asociadas al menos a algún tipo de impacto que casi no tienen costo. La única intervención que se mantiene es la número 2 (asignar a los maestros más destacados al primer grado), que pese a tener un costo muy bajo, según los expertos tendría un fuerte impacto.

Otra intervención que cumple esta condición es la de hacer cumplir las regulaciones que definen la duración del año escolar oficial, pese a que podría acarrear complicaciones políticas, especialmente en países donde las huelgas del magisterio son comunes. Imponer la prohibición de cambiar de curso al profe-

\section{CUADRO 6 \\ Intervenciones con el índice de costo- efectividad esperado más alto}

Intervención

Indice de costo-efectividad

2. Asignar a los mejores maestros al primer grado

1531.2

3. Asegurar el cumplimiento de la duración oficial del año escolar

699.6

1. Política que prohíba cambiar al profesor de curso durante el año

13. Administrar pruebas a un $10 \%$ de los estudiantes de $4^{\circ}$ grado y distribuir los resultados entre los maestros

11. Descentralización (sin fortalecer la supervisión)

27. Campañas a través de los medios para que los padres den estimulación temprana y compartan lecturas con sus hijos 
sor durante el año escolar tampoco implica mayores costos, pero puede ocasionar problemas administrativos. El retiro de un maestro durante el año escolar exigiría su reemplazo por un substituto temporal, como alternativa a transferir un maestro de otra escuela.

El costo de administrar pruebas a muestras de alumnos es bastante más bajo que el costo de hacerlo a través de muestreos por universos. Para que esta última modalidad fuera práctica, los resultados debieran ser presentados en una forma amistosa al usuario e incluir sugerencias de mejoramiento.

En opinión de los expertos, la centralización de la administración educativa en la región se encuentra tan "osificada" que descentralizarla, incluso sin el fortalecimiento de una autoridad supervisora, debiera tener un efecto positivo, ya que prácticamente no tiene costo. Por último, aparentemente las campañas a través de los medios de comunicación tienen un costo relativamente bajo, pero pueden tener un impacto considerable.

El cuadro 7 muestra las intervenciones que tienen el valor costo-efectividad más bajo. Obviamente, los programas de alimentación escolar son de alto costo y su efecto en el aprendizaje posiblemente sería sólo marginal; pero podrían influir sustancialmente en otros parámetros, como la asistencia, la salud y la distribución del ingreso. Asimismo, los aumentos de sueldo modestos que no vayan acompañados de mayores responsabilidades no constituyen un enfoque eficiente (en términos de costo). Por último, en la actualidad, las computadoras tampoco representarían una alternativa eficiente para la educación primaria.

CUADRO 7

\section{Intervenciones con el menor índice de costo-efectividad esperado (En porcentajes)}

Intervención

Indice de costo-efectividad

22. Programas de alimentación escolar (almuerzo gratuito para el $100 \%$ del alumnado)

0.2

39. Acceso a computadoras 1 hora a la semana

23. Programas de alimentación escolar (almuerzo gratuito para el $50 \%$ del alumnado)

20. Programas de alimentación escolar (colación gratuita para el 50\% del alumnado) 0.3

31. Capacitación en servicio para docentes (sin material de seguimiento)

8. Aumento del sueldo de los profesores del orden del $20 \%$

\section{$\mathrm{X}$}

\section{Comparación de estos resultados con estimaciones de planificadores de América Latina}

Cuando se comparan las estimaciones del panel de expertos con las de los planificadores y asesores de los ministerios de educación latinoamericanos (que asistieron a los cursos de planificación organizados por la UNESCO en 1994 y 1996), se observa que éstos son más optimistas que los expertos sobre el impacto que podrían tener las intervenciones (en promedio, 19\% contra 10\%). Es posible que los primeros no estén suficientemente familiarizados con la literatura sobre el tema de la efectividad de las intervenciones, la cual suele ser muy conservadora (Schiefelbein, Wolff y Schiefelbein, 1998). Por otra parte, en las opiniones sobre la probabilidad de una implementación exitosa, ocurrió exactamente lo contrario (48\% contra $63 \%$ ). En particular, los planificadores se inclinaron menos por las implementaciones que necesitan un mayor financiamiento, y finalmente estimaron que cada intervención aumentaría el costo unitario en un $14 \%$ como promedio, casi tres veces el porcentaje calculado por los autores (5\%).

En cuanto a los cálculos de costo-efectividad, los valores obtenidos por los planificadores y los expertos son del mismo orden (cuando se comparan usando las estimaciones de costo del anexo), aunque exhiben algunas diferencias. Los planificadores atribuyen a la descentralización $\left(\mathrm{N}^{\circ} 11\right)$, a la prohibición de cambiar al profesor del curso $\left(\mathrm{N}^{\circ} 1\right)$, a las mejoras en el área de capacitación inicial del docente $\left(\mathrm{N}^{\circ} 35\right)$, a las modificaciones curriculares $\left(\mathrm{N}^{\circ} 36\right)$, a los programas pre- 
escolares $\left(\mathrm{N}^{\circ} 29\right.$ y 30$)$, a las iniciativas tradicionales de capacitación en servicio $\left(\mathrm{N}^{\circ} 31\right)$ y al uso de computadoras ( $\left.\mathrm{N}^{\circ} 39\right)$, un costo-efectividad apreciablemente mayor (más del doble) que los expertos. Por su parte, los expertos atribuyeron un mayor costo-efectividad sólo a aquellas intervenciones que involucran la reduc- ción del aparato burocrático $\left(\mathrm{N}^{\circ} 9\right)$, programas televisivos para preescolares y campañas a través de los medios de comunicación ( $\mathrm{N}^{\circ} 26$ y 27), la extensión del día y año escolar ( $\mathrm{N}^{\circ} 4$ y $\left.\mathrm{N}^{\circ} 5\right)$, el sobresueldo para maestros rurales $\left(\mathrm{N}^{\circ} 6\right)$ y el aumento de salarios del profesorado ( $\mathrm{N}^{\mathrm{os}} 7$ y 8 ).

\section{XI}

\section{Conclusiones de las estimaciones de costo-efectividad para las 40 estrategias}

El índice de costo-efectividad es una herramienta diseñada para "definir conjeturas en forma explícita", y los coeficientes analizados tienen las limitaciones (y fortalezas) de los expertos que entregaron sus estimaciones. Su principal valor está dado por la ayuda que ofrece para formular preguntas importantes sobre los componentes que incluyen los proyectos examinados. Llega en un momento muy oportuno para América Latina, dados el creciente consenso sobre el papel fundamental que desempeña la educación en el éxito económico y social, el interés de los Presidentes expresado en el acuerdo suscrito en la Cumbre de Jefes de Estado en 1998, el gran aumento de la inversión en el campo de la educación realizada por los gobiernos y organismos internacionales en los años noventa y los escasos impactos que muestran los informes de la OCDE (2000) y la UNESCO (2000).

La necesidad de definir estrategias en el ámbito educacional no es privativa de quienes se desempeñan en este campo, sino que también interesa a los líderes políticos y de la industria. Quizás el sencillo instrumento elaborado pueda ayudar a quienes deben tomar decisiones a distinguir entre las estrategias que "es posible esperar que funcionen" y las que "no es fácil que lo hagan", y contribuir a fortalecer el consenso social sobre la necesidad de invertir eficientemente en el sector de la educación.

El ejercicio realizado también es un excelente método de enseñanza para elaboradores de políticas y planificadores, ya que los obliga a hacer explícito su pensamiento. Una forma óptima de operar consiste en crear pequeños grupos que trabajen con alrededor de cinco intervenciones. La idea es trabajar en conjunto y presentar las conclusiones ante una sesión plenaria. Se reducen así las incongruencias, ambigüedades y contradicciones sobre el costo-efectividad del proyecto o la estrategia.

En general, muchos de los programas y proyectos puestos en marcha en América Latina no coinciden con las recomendaciones derivadas de este ejercicio. En particular, los enfoques simples y de bajo costo, como la asignación de maestros, la continuidad del docente y las campañas a través de los medios de comunicación, no suelen quedar incluidos en los proyectos. Lamentablemente, son muy escasas las intervenciones que, con el apoyo de la información empírica, muestran avances importantes en el nivel de aprendizaje.

Lo anterior es particularmente cierto en el caso de intervenciones actualmente en boga, como la descentralización, la administración de pruebas y el uso de computadoras. Los errores son costosos, y en momentos en que la educación es aclamada como factor clave del desarrollo económico y social, y se hacen en ella grandes inversiones regionales y globales, es indispensable reexaminar el impacto de las diversas intervenciones.

El hecho de haber contado con la participación de no más de diez expertos, aunque sin duda del máximo nivel, puede reducir la confiabilidad de las respuestas: si sólo uno de ellos mostrara una predisposición excepcional, el efecto sobre el promedio general sería significativo. Los autores han identificado a otros expertos y recomiendan que, más adelante, se lleven a cabo nuevas encuestas con la participación de 20 a 25 expertos. Ejercicios similares pueden realizarse para la educación media y superior. 


\section{XII \\ Recomendaciones que emergen del examen de los resultados}

El ejercicio sugiere cuatro recomendaciones sobre las políticas asociadas a intervenciones educativas:

i) Efectuar aquellas intervenciones de "gran impacto en el rendimiento", particularmente las relacionadas con intervenciones múltiples, materiales didácticos y apoyo diferencial a la educación rural, que impliquen costos moderados. Dado su considerable efecto potencial $\mathrm{y}$, a pesar de sus costos, estas intervenciones debieran llevarse a cabo. Sin embargo, es preciso tener cautela ante posibles problemas de aplicación y desarrollo.

ii) Poner en marcha intervenciones que impliquen un costo muy bajo y que tengan un impacto positivo. Estas son las que suelen pasar inadvertidas (por ejemplo, regular la duración del año escolar o asignar buenos maestros al primer grado).

iii) Evitar aquellas intervenciones que son de alto costo y que por sí solas (sin actividades u objetivos suplementarios) no generan un impacto importante, es decir, no constituyen una buena inversión. Esto se aplica particularmente al aumento de salarios, la utilización de computadoras y los programas de alimentación escolar.

iv) Evaluar los proyectos propuestos para mejorar la educación del país con las estimaciones del panel de expertos y justificar las diferencias. El disponer de un conjunto de estrategias sobre las cuales han opinado los expertos permite, al menos, madurar una justificación detallada de tales diferencias.

En las preguntas que se formulen en el futuro convendría hacer explícitos los costos y efectos esperados, a fin de dar mayor transparencia a las conjeturas de quienes propongan las estrategias acerca de aquellos aspectos más debatibles, las que en ciertas circunstancias pueden o no funcionar. Ejercicios de este tipo pueden alertar oportunamente a los elaboradores de la política educativa acerca del valor relativo de las estrategias que han adoptado, permitiéndoles reexaminar sus presunciones. El cálculo del costo de las intervenciones es en sí de gran utilidad, ya que no suele hacerse en forma sistemática. Por ende, estas estimaciones pueden ser utilizadas para evaluar, o al menos proporcionar una cifra de referencia, con miras a calcular el costo de los componentes de estos proyectos en diversos países.

Actualmente, América Latina tiene una gran oportunidad de realizar investigación aplicada, ya que todos los países de la región están realizando evaluaciones a escala nacional, ya sea a través de muestras o de universos de alumnos, y estarán ahora en condiciones de aprovechar tal investigación como instrumento que les ayude a identificar las estrategias que generen los mejores resultados de aprendizaje. 


\begin{tabular}{|c|c|c|}
\hline Intervención & $\begin{array}{l}\text { Aumento del } \\
\text { costo unitario }(\%)\end{array}$ & Explicación del cálculo de costo \\
\hline 1 & 0.01 & Costo nominal del orden de los 30 mil dólares. \\
\hline 2 & 0.01 & Costo nominal del orden de los 30 mil dólares, por información y control. \\
\hline 3 & 0.01 & Costo nominal del orden de los 30 mil dólares, por proveer información y garantizar cumplimiento. \\
\hline 4 & 15.0 & $\begin{array}{l}\text { Un aumento del } 16.7 \% \text { en horas, equivale a un aumento de sueldos del } 16.7 \% \text { (90 \% del costo } \\
\text { total). } 16.7 \% \text { x } 90 \%=15 \% \text { o } 30 \text { dólares por alumno. }\end{array}$ \\
\hline 6 & 13.5 & $\begin{array}{l}\text { El } 30 \% \text { de los estudiantes son de zonas rurales. Para este grupo, el aumento salarial equivale al } 50 \% \\
\text { del } 90 \% \text { del costo unitario ( } 0.45 \text { x } 200 \text { dólares) o } 90 \text { dólares por estudiante del grupo beneficiario. } \\
\text { Para este sistema, el costo es de } 27 \text { dólares por estudiante. (El mayor costo total es } 90 \text { x } 600000 \text { con } \\
\text { relación al total de } 400 \text { millones de dólares). }\end{array}$ \\
\hline 7 & 9.0 & El aumento equivaldría a 18 dólares por estudiante. \\
\hline 8 & 18.0 & El aumento equivaldría a 36 dólares por estudiante. \\
\hline 9 & 0.3 & $\begin{array}{l}\text { La burocracia representa el } 5 \% \text { del presupuesto total, o } 20 \text { millones de dólares, que equivale a } 10 \\
\text { dólares por estudiante. Un } 50 \% \text { de reducción en el número de burócratas reduce el costo a la mitad } \\
\text { ( } 10 \text { millones de dólares). Las mejoras salariales incrementan el costo en } 10.5 \text { millones de dólares. } \\
\text { El aumento equivale a } 500000 \text { dólares. También puede ser calculado directamente sobre la base del } \\
\text { costo unitario. El costo actual es de } 10 \text { dólares. Si la burocracia se reduce a la mitad, el nuevo costo } \\
\text { unitario es de } 5 \text { dólares; si el costo se incrementa } 2.1 \text { veces, el costo unitario se eleva a } 10.5 \text { dólares. } \\
\text { El aumento total del costo unitario es de } 50 \text { dólares. }\end{array}$ \\
\hline 10 & 0.3 & SIG estimado en 1000000 de dólares o 0.50 dólares por estudiante. \\
\hline 11 & 0.1 & $\begin{array}{l}\text { Dado que no se ha considerado ampliar la facultad del Ministerio de Educación para fiscalizar y } \\
\text { evaluar, se estima que el costo no excedería de } 300000 \text { dólares o } 0.15 \text { dólares por concepto de } \\
\text { folletos para rectores y centros de padres, más la implementación de un sistema de información y } \\
\text { difusión. }\end{array}$ \\
\hline 12 & 1.3 & $\begin{array}{l}\text { El costo de mejorar el flujo de información y de ampliar la facultad de fiscalización y evaluación, } \\
\text { reforzando la administración de pruebas, la producción de estadísticas y la gestión financiera, es de } \\
\text { aproximadamente } 3 \text { millones de dólares. Administrar pruebas a } 330 \text { mil alumnos de } 4^{\circ} \text { grado tiene } \\
\text { un costo de } 5 \text { dólares por estudiante; otros costos incluyen cerca de } 1000000 \text { de dólares para un } \\
\text { MIS y costos varios del orden de } 300000 \text { dólares. El costo unitario total es de } 2.65 \text { dólares. }\end{array}$ \\
\hline 13 & 0.1 & $\begin{array}{l}\text { Una batería apropiada de pruebas tiene un costo aproximado de } 5 \text { dólares por alumno. Sin embargo, } \\
\text { sólo un } 10 \% \text { de los alumnos de } 4^{\circ} \text { grado es evaluado. El } 4^{\circ} \text { grado representa } 1 / 6 \text { del alumnado total, } \\
\text { por lo tanto, sólo un } 1.67 \% \text { de los estudiantes rinde la prueba. Para dichos estudiantes el costo es } \\
\text { de } 5 \text { dólares; para el sistema el costo es de } 0.08 \text { dólares. la distribución de resultados entre los maestros } \\
\text { de } 4^{\circ} \text { grado agrega } 0.02 \text { dólares, para un total de } 0.10 \text { dólares. }\end{array}$ \\
\hline 14 & 0.4 & $\begin{array}{l}\text { El costo del seminario de seguimiento para la totalidad de los maestros de } 4^{\circ} \text { grado, equivale a una } \\
\text { semana de trabajo docente. El costo unitario es de } 1 / 6 \text { de } 4.50 \text { dólares o } 0.75 \text { dólares, más } 0.10 \text { dó- } \\
\text { lares por administración de pruebas. El costo total del sistema es de } 0.85 \text { dólares. }\end{array}$ \\
\hline 15 & 0.8 & $\begin{array}{l}\text { Incluye una semana de capacitación. Se administran pruebas a la totalidad de los alumnos de } 4^{\circ} \\
\text { grado. El costo es } 1 / 6 \text { de } 5.00 \text { dólares o } 0.83 \text { dólares, más } 0.75 \text { dólares por concepto de capacitación. } \\
\text { El costo total es de } 1.58 \text { dólares. }\end{array}$ \\
\hline 16 & 1.5 & $\begin{array}{l}\text { Debe establecer que se proporcionan dos libros de texto (español y matemáticas). Se asume } 1.50 \\
\text { dólares por libro. Total } 3.00 \text { dólares por estudiante. }\end{array}$ \\
\hline 17 & 3.8 & $\begin{array}{l}\text { Se asume que el salario del maestro es } 90 \% \text { del costo unitario de } 200 \text { dólares, vale decir, } 180 \text { dólares } \\
\text { por estudiante. Dividido por } 40 \text {, una semana de trabajo del docente equivale a } 4.50 \text { dólares por alumno. } \\
\text { Si a esto le sumamos } 3 \text { dólares (intervención 16), el total es de } 7.50 \text { dólares. }\end{array}$ \\
\hline 18 & 1.5 & $\begin{array}{l}\text { El costo de impresión es de } 8.75 \text { dólares (cuatro libros de texto); el costo de preparación es de } 500000 \\
\text { dólares, vale decir, } 0.25 \text { dólares por estudiante. El costo total es de } 9.00 \text { dólares. Los libros tienen } \\
\text { una vida útil de } 3 \text { años, lo que se traduce en un costo de } 3.00 \text { dólares. }\end{array}$ \\
\hline 19 & 1.4 & $\begin{array}{l}\text { Se asume un precio (por grandes cantidades) de } 2.00 \text { dólares por libro, de modo que la biblioteca } \\
\text { tendría un costo de } 400 \text { dólares. Proyectado a } 5 \text { años el costo se reduce a } 80 \text { dólares. Considerando } \\
29 \text { estudiantes por clase el costo unitario equivaldría a } 275 \text { dólares. }\end{array}$ \\
\hline 20 & 13.5 & $\begin{array}{l}\text { Se calcula el costo de la leche en } 0.10 \text { dólares y del pan en } 0.05 \text { dólares. El costo total es } 0.15 \text { dólares } \\
\text { x } 180 \text { días, o } 27 \text { dólares por estudiante. }\end{array}$ \\
\hline 21 & 6.8 & $\begin{array}{l}\text { Igual que arriba, salvo que sólo lo recibe la mitad del alumnado. Luego, el costo unitario es de } 13.50 \\
\text { dólares. }\end{array}$ \\
\hline 22 & 36.0 & $\begin{array}{l}\text { El costo diario estimado para almuerzos es de } 0.40 \text { dólares; por lo tanto, el costo es de } 0.40 \text { dólares } \\
\text { x } 180 \text { o } 72 \text { dólares por estudiante. }\end{array}$ \\
\hline 23 & 18.0 & La mitad del costo de la intervención 22 , vale decir, 36 dólares. \\
\hline
\end{tabular}


Anexo 1 (continuación)

\begin{tabular}{lcc}
\hline Intervención & $\begin{array}{c}\text { Aumento del } \\
\text { costo unitario }(\%)\end{array}$ & Explicación del cálculo de costo
\end{tabular}

No incluye servicios médicos prestados por el sistema de salud. Un facultativo puede examinar a 28 alumnos al día, durante 180 días, lo que equivale a 5 mil estudiantes al año. El sueldo del médico es de 24000 dólares, lo que resulta en un costo unitario de 4.80 dólares.

Sólo detección, si bien los alumnos con afecciones a la vista pueden ocupar los asientos de adelante. Con una pequeña cantidad de dinero y algunos materiales, el maestro podría tomar esta iniciativa. Se ponen a disposición 250 programas de televisión para su uso en el hogar. El costo absoluto estimado es de 3000000 de dólares, asumiendo la compra de programas de gran prestigio como Plaza Sésamo.

El costo total de preparación y compra de espacios publicitarios en televisión se calcula en 500000 , vale decir, 0.25 dólares por estudiante.

El costo es de 200 dólares para el $50 \%$ de los estudiantes prorrateado a lo largo de 6 años; vale decir, 33.33 dólares para el grupo beneficiario y 16.67 dólares por estudiante considerando todo el sistema.

La mitad del costo; vale decir, 16.67 dólares para el grupo beneficiario y 8.34 dólares por estudiante considerando todo el sistema.

Proporcionado a un $50 \%$ del alumnado. El costo es igual al calculado para la intervención 29.

Cuatro semanas completas de perfeccionamiento docente, equivalen a 4.50 dólares x $4=18.0$ dólares. Si a esto se le suma el costo de la preparación del curso, el material y los gastos de traslado, el costo total se aproxima a 20 dólares.

Una semana de capacitación se calcula a 4.50 dólares, igual al caso anterior.

Igual al caso anterior.

Igual al caso anterior.

El programa de subsidio se calcula en 200 dólares por docente graduado (5 x 4) que enseña durante 20 años. Luego, el costo es de 20 dólares por año. La relación maestro/estudiante es 1:29, por consiguiente el costo anual es 20/29 o 0.70 dólares. (Otra forma de calcular este costo es considerar que cada año se capacitan 7 mil maestros para reemplazar al $10 \%$ de un magisterio de 70 mil. Siete mil maestros nuevos x 200 dólares $=1400000$ dólares, o un costo unitario de 0.70 dólares).

Basado en la contratación de expertos locales y la distribución de manuales curriculares, no en estudios de investigación técnica. Se estima un costo de 400000 dólares para cubrir esencialmente el costo de los expertos locales y un costo de distribución de los manuales muy bajo (1 dólar por manual, para un total de 70 mil copias).

El currículo bilingüe tiene un costo fijo bajo de aproximadamente 100000 dólares, asociado con la contratación de profesores bilingües. Esto corresponde a 0.50 dólares por alumno atendido (un $10 \%$ de los estudiantes). Se deben suministrar los libros necesarios y capacitar a los maestros durante por lo menos una semana al año. Por consiguiente, el costo variable para el $10 \%$ de la población es de 4.50 dólares, por la semana de capacitación, más 3 libros a 2 dólares (por un total de 6 dólares); el total para alumnos indígenas es aproximadamente de 10.55 dólares. El costo para la totalidad del sistema equivale al $10 \%$, o 1.05 dólares.

500000 dólares por concepto de preparación, o 0.25 dólares por estudiante, sin el uso de asistencia técnica extranjera (caso de Venezuela). El costo de la radio (29 dólares por aparato por clase) es aproximadamente 1 dólar por estudiante, si bien su duración se estima en 3 años; luego, su costo es de 0.33 dólares. El costo de los materiales es aproximadamente de 0.50 dólares. El costo unitario total es de 1.08 dólares.

Se estima un costo de 2000 dólares en computadoras más 100 dólares para otras modificaciones. Las computadoras tienen una vida útil de 4 años; por lo tanto, su costo anual es del orden de 525 dólares. Las computadoras son utilizadas por 30 estudiantes a razón de 1 hora por semana (30 horas semanales) o 16.50 dólares por estudiante. A este costo se debe sumar el de un maestro a tiempo completo que trabaje 27 horas semanales (una vez a la semana un curso trabaja con dos maestros por un período). El costo del educador representa el 1/27 del $90 \%$ del costo unitario, o 6.67 dólares. El mantenimiento de las computadoras equivale a 200 dólares por año, o 6.67 dólares adicionales por estudiante. El total por estudiante es de 29.84 dólares. La compra de computadoras en desuso por 1000 dólares, reduciría drásticamente dicho costo y, a su vez, bajaría a la mitad el costo de mantenimiento y seguridad. Otra opción es contratar a un técnico a $2 / 3$ del costo del maestro. El costo total podría verse reducido a 8.25 dólares +5 dólares +3.33 dólares $=16.58$ dólares, a pesar que esto último puede no ser muy factible.

El costo está basado en las siguientes estimaciones previas: libros de texto 3 dólares; materiales didácticos de autoaprendizaje 3 dólares; una semana de capacitación 4.50 dólares; administración local de la escuela 2.65 dólares; sistema de evaluación 0.85 dólares. El costo total es de 14 dólares.

a El costo total del sistema primario es del orden de 400 millones de dólares y el costo unitario es 200 dólares. 


\section{Bibliografía}

Araujo Oliveira, J. B. (1998): A Pedagogia du Sucesso, Brazil, Belo Horizonte.

Banco Mundial (1994): Priorities and Strategies for Education, Washington, D.C.

Carnoy, M. y C. de M. Castro (1997): La reforma educativa en América Latina: Actas de un seminario, Washington D.C., Banco Interamericano de Desarrollo (BID).

Castillo, M. (1998): La descentralización de los servicios de educación en Nicaragua, serie Reformas de política pública, $\mathrm{N}^{\circ}$ 53, Santiago de Chile, Comisión Económica para América Latina y el Caribe (CEPAL).

Chrispeels, J. (1997): Educational policy implementation in a shifting political climate: The California experience, American Education Research Journal, vol.34, $\mathrm{N}^{\circ} 3$.

El Salvador, Ministerio de Educación (1996): Los educadores y la comunidad, San Salvador.

Fuller B. y P. Clarke (1994): Raising schools effects while ignoring culture? Review of Economic Research vol 64, $\mathrm{N}^{\circ} 1$.

Gutman, C. (1993): Todos los niños pueden aprender. El Programa de las 900 Escuelas para los sectores pobres de Chile, París, Organización de las Naciones Unidas para la Educación, la Ciencia y la Cultura (UNESCO).

Harbison, R. y E. Hanushek (1992): Educational Performance of the Poor: Lessons from Rural Northeast Brazil, Nueva York, Banco Mundial.

Hornik, R., H. Ingle, M., Macanany y W. Schramm (1973): Television and Educational Reform in El Salvador, Stanford, California, Stanford University.

Jamison, D., B. Searle, S. Heyneman y K. Galda (1981): Improving Elementary Mathematics Education in Nicaragua: An Experimental Study of the Impact of Textbooks and Radio on Achievement, Documento de trabajo, $\mathrm{N}^{\circ}$ 5, Washington, D.C, Banco Mundial.

Lockheed M.. y A. Verspoor (1991): Improving Primary Education in Developing Countries, Nueva York, Banco Mundial.

Meza, D. (1997): Descentralización educativa, organización y manejo de las escuelas a nivel local: El caso de El Salvador, Informe LAC, $\mathrm{N}^{\circ}$ 9, Washington, D.C., Banco Mundial/Educación con Participación Comunal (EDUCO).
McEwan, P. (1995): Primary School Reform for Rural Development: An Evaluation of Colombian New Schools, Washington, D.C., Banco Mundial.

OCDE (Organización de Cooperación y Desarrollo Económicos) (2000): Literacy in the Information Age: Final report of the International Adult Survey, Canadá, Statistic Canada.

Psacharopoulos, G., C. Rojas y E. Vélez (1995): Achievement evaluation of Colombia's Escuela Nueva: Is multigrade the answer? Comparative Education Review, vol. 37, $\mathrm{N}^{\circ} 3$.

Reimers, F.y N. McGinn (1997): Informed Dialogue. Using Research to Shape Education Policy Around the World, Wesport, Connecticut, Praeger.

Rojas, C. y Z. Castillo (1998): Evaluación del Programa Escuela Nueva en Colombia, Santafé de Bogotá, Instituto SER de Investigación.

Rojas, C. y M.Esquivel (1998): Assessment Programs in Latin America, Documento de trabajo, Washington, D.C., Banco Mundial.

Schiefelbein, E. (coord.) (1998): Educación en las Américas: calidad y equidad en el proceso de globalización, Washington, D.C., Organización de los Estados Americanos (OEA).

Schiefelbein, E., L. Wolff y P. Schiefelbein (1998): Cost-Effectiveness of Education Policies in Latin America: A Survey of Expert Opinion, Washington D.C.

Swope, J. y M. Latorre (1998): Fe y Alegría Schools in Latin America Educational Communities were the Pavements, End, Santiago de Chile, LOM Ediciones.

UNESCO (1998): Primer estudio internacional comparativo, Santiago de Chile, Laboratorio Latinoamericano de Evaluación de la Calidad de la Educación.

- (2000): Alfabetismo funcional en siete países de América Latina, Santiago de Chile.

Verspoor, A. (1989): Pathways to Change: Improving the Quality of Education in Developing Countries, Documento de Trabajo, Washington, D.C., Banco Mundial.

Wolff, L., E. Schiefelbein y J. Valenzuela (1994): Improving the Quality of Primary Education in Latin America and the Caribbean, Washington, D.C., Banco Mundial. 